\title{
Archéopages
}

Archéopages

Archéologie et société

Hors-série 2 | 2010

Archéologie sans frontières

\section{Perspectives pour la coopération archéologique franco-égyptienne}

\section{Guillemette Andreu-Lanoë}

\section{(2) OpenEdition}

1 Journals

Édition électronique

URL : https://journals.openedition.org/archeopages/802

DOI : 10.4000/archeopages.802

ISSN : 2269-9872

\section{Éditeur}

INRAP - Institut national de recherches archéologiques préventives

\section{Édition imprimée}

Date de publication : 1 octobre 2010

Pagination : 107-109

ISSN : 1622-8545

\section{Référence électronique}

Guillemette Andreu-Lanoë, «Perspectives pour la coopération archéologique franco-égyptienne », Archéopages [En ligne], Hors-série 2 | 2010, mis en ligne le 01 octobre 2010, consulté le 23 février 2023. URL : http://journals.openedition.org/archeopages/802 ; DOI : https://doi.org/10.4000/ archeopages. 802 


\section{Perspectives pour la coopération archéologique franco-égyptienne}

Guillemette Andreu-Lanoë

Musée du Louvre

$\mathrm{D}$ epuis les années 1950, qui ont vu la chute du roi Farouk et l'arrivée du nassérisme à la tête de l'Égypte, les pratiques quotidiennes, culturelles et patrimoniales de l'Égypte n'ont cessé d'évoluer. En soixante ans, le pays est passé de moins de 30 millions d'habitants à plus de 70 millions. Les fellahin, ces paysans en gallabiyeh qui renvoyaient une image éternelle de l'Égypte avec leurs ânes et leurs bufflesses, sillonnent désormais la campagne sur des engins mécanisés et diversifient leur production, autrefois axée essentiellement sur le coton et les céréales. L'économie vit de plus en plus du tourisme, dont le potentiel s'enrichit de découvertes archéologiques spectaculaires qui occupent régulièrement la une des médias. Les vestiges pharaoniques s'offrent à la visite des touristes comme un produit presque ordinaire, tant la vallée du Nil peut en présenter. L'Égypte a le privilège, unique à une telle échelle, de posséder un patrimoine d'une abondance éblouissante, enrichi de découvertes considérables depuis la fin du $\mathrm{XIX}^{\mathrm{e}}$ siècle, tandis que les explorations archéologiques qui s'y poursuivent révèlent chaque année des «trésors » insignes et une impressionnante moisson de données.

Alors que la culture arabe traditionnelle puis nassérienne avait détourné les Égyptiens de leur passé pré-islamique, la situation a évolué ces quinze dernières années. Les sites et les musées reçoivent des groupes scolaires, sous la houlette de conférenciers égyptiens compétents, qui ont le talent nécessaire pour sensibiliser les jeunes classes à leur patrimoine. Des voyages de découverte des temples du sud du pays sont proposés aux élèves cairotes durant les vacances scolaires, transformant de façon charmante et bruyante la majestueuse salle hypostyle de Karnak en vaste cour de récréation.

Mais la réappropriation du patrimoine pharaonique n'est évidemment pas l'objectif prioritaire de l'État égyptien, qui doit gérer une explosion démographique galopante. Dans toutes les grandes villes qui sont aussi des cités antiques - Alexandrie, Assiout, Assouan, Héliopolis, Le Caire, Louxor, Minia, etc. -, des travaux publics ou privés de grande envergure détruisent de nombreux sites d'un intérêt scientifique majeur. Ainsi, dans le delta oriental, sur le site de Tell-el Maskhouta, Mohamed Abdel Maksoud a très récemment sauvé une tombe aux décors uniques, que des travaux d'aménagement avaient mise au jour et risquaient de détruire. Cette tombe se situe au pied d'un immeuble d'habitations collectives, à proximité d'une voie ferrée, d'une route et d'un canal. On imagine sans peine que ces derniers ont été bâtis, sans scrupules, au détriment d'autres vestiges, car il est rare que l'on trouve une tombe isolée. Parfois, c'est aussi la beauté d'un paysage naturel qui est touchée par le site management systématique que les chefs des gouvernorats mettent en place. Une autoroute à quatre voies relie ainsi aujourd'hui sur $4 \mathrm{~km}$ le parvis du temple de Karnak à la ville de Louxor, mettant à mal le charme légendaire des rives du Nil en ces lieux.

Pays en développement, l'Égypte pratique encore peu l'archéologie préventive. C'est sans doute dans cette direction que l'archéologie française en Égypte doit se positionner, afin d'accompagner les Égyptiens dans la protection, l'étude et la mise en valeur de leur patrimoine. La réflexion qui s'impose d'ailleurs aux missions œuvrant sur le sol égyptien, quelle que soit leur nationalité, porte sur le débat - le choix pourrait-on dire -, entre valorisation $\mathrm{du}$ patrimoine et recherche de nouveaux vestiges, toujours prêts à émerger sous les truelles des fouilleurs.

Aujourd'hui, le comité permanent du Conseil suprême des antiquités égyptiennes dirigé par Zahi Hawass - récemment nommé vice-ministre n'accorde qu'au compte-gouttes les permis de fouilles dans les sites prestigieux, tels que la nécropole thébaine ou la zone des pyramides. Une équipe voulant y ouvrir un chantier doit pouvoir invoquer un enjeu scientifique majeur. En revanche, partout où les vestiges sont en danger, le même comité accorde aisément son autorisation et encourage les archéologues à s'investir au mieux dans la récolte des données en perdition. De la part des autorités égyptiennes, la prise de conscience du péril qui menace ces sites est chaque année plus perceptible. De ce fait, les zones archéologiques prioritaires sont le nord du Sinaï et le delta du Nil, où de nombreux sites fragilisés par des mises en cultures massives et une irrigation intensive méritent d'être explorés avant les autres.

Face à cette évolution, l'égyptologie et l'archéologie françaises doivent s'assigner ces objectifs nouveaux et inscrire dans leurs programmes la participation au sauvetage des sites et vestiges menacés. De nombreux égyptologues et archéologues d'autres pays sont déjà engagés dans cette direction, puisqu'ils mènent des chantiers en dehors des sites prestigieux, où ils recueillent nombre d'informations essentielles qui ne concernent pas l'élite pharaonique. Si elle veut augmenter à terme son efficacité, l'égyptologie mondiale doit se doter d'un solide fer de lance scientifique égyptien. La patrie de Champollion peut y contribuer puisque plus de 200 chercheurs français travaillent à restituer le passé égyptien. Il serait donc logique que des bourses doctorales facilitent la transmission du savoir vers la jeune génération égyptienne. Cette transmission peut aussi se faire en allant enseigner en Égypte, où le métier d'égyptologue et d'archéologue, incarné par Zahi Hawass, a gagné des lettres de noblesse dans la société. Récemment, un accord entre l'Université française d'Égypte et l'Institut national du patrimoine a été conclu pour former les Égyptiens dans le domaine de la gestion du patrimoine. La France, avec notamment le musée du Louvre, est par ailleurs bien placée pour les techniques de conservation et de restauration. Puisque l'évolution de l'archéologie égyptienne 

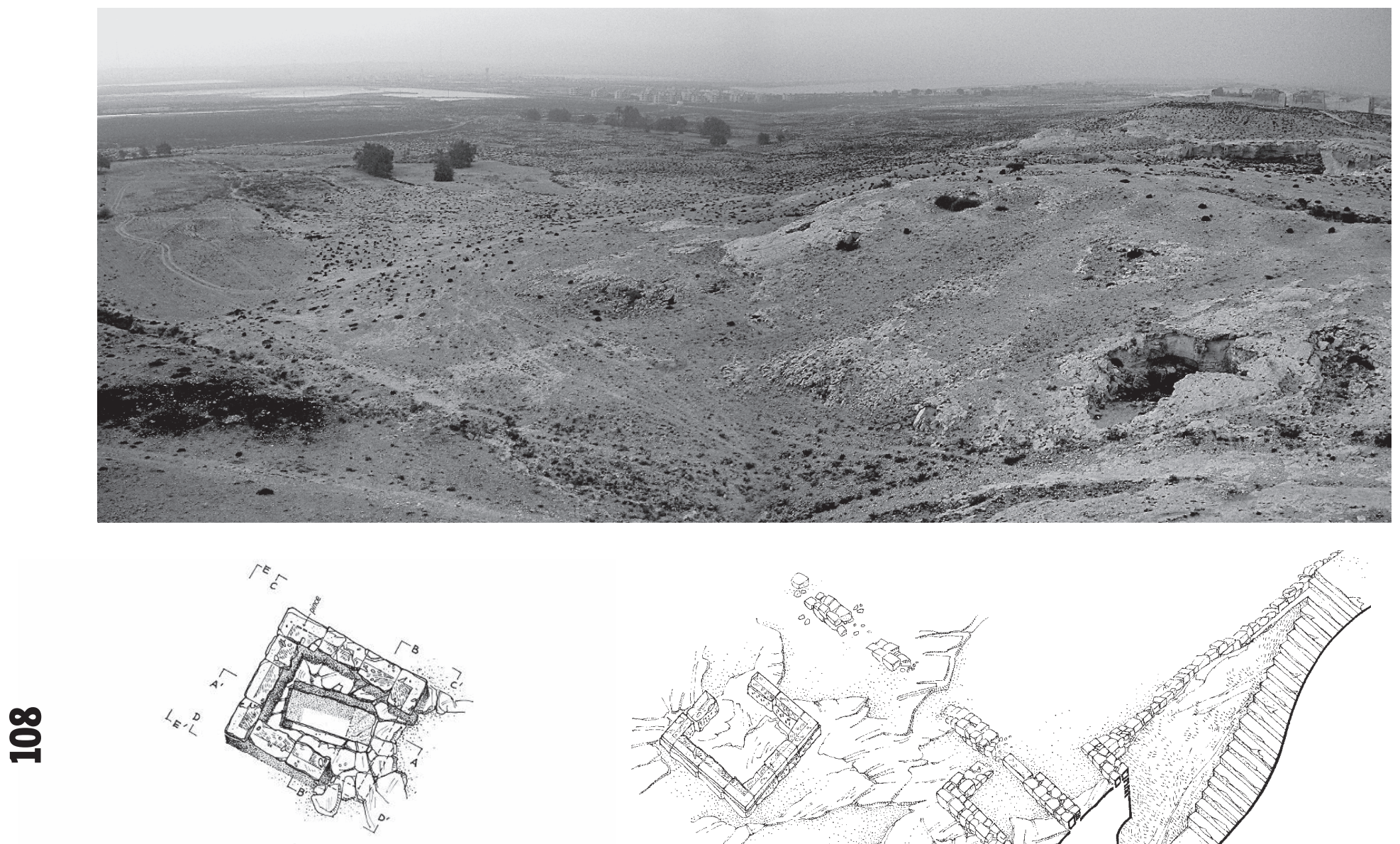

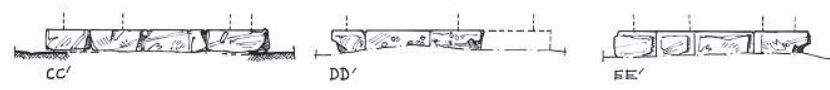
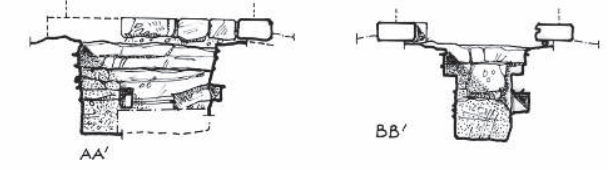

i

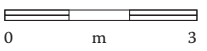

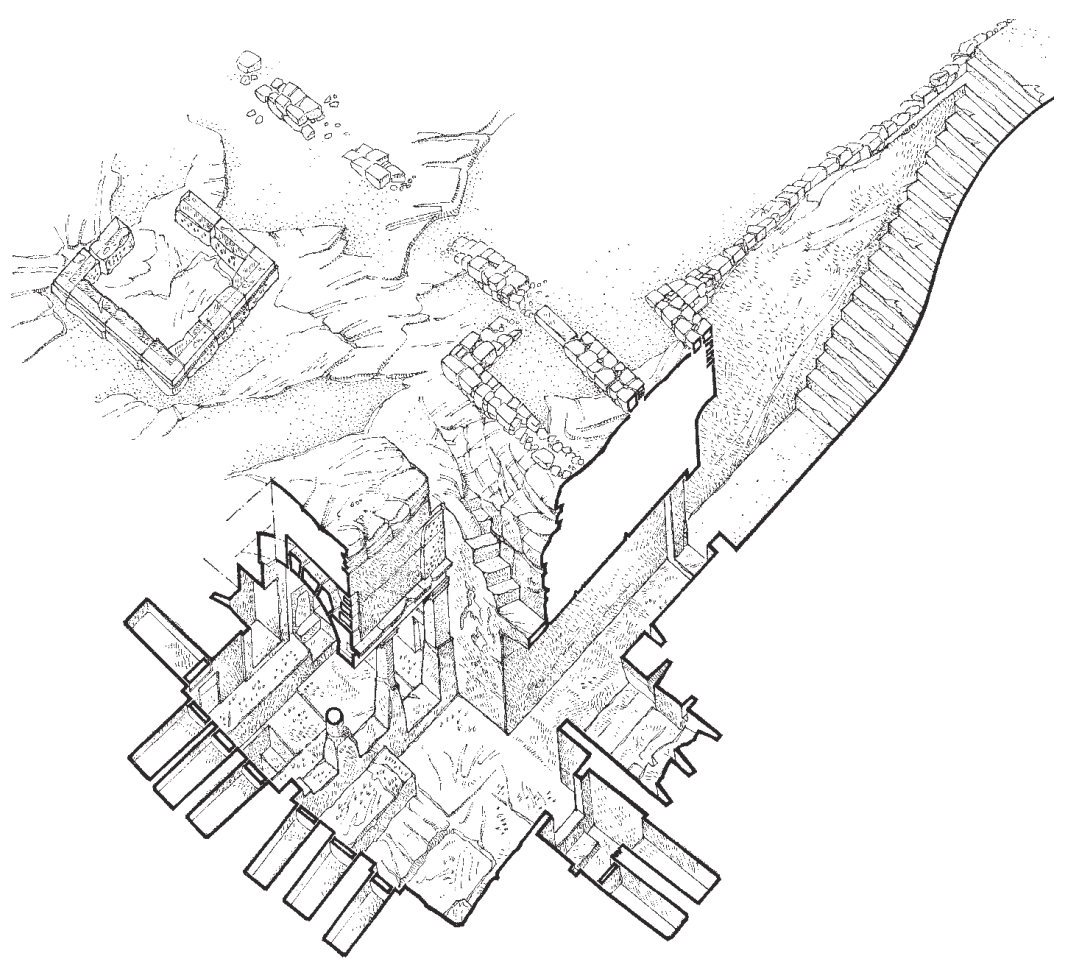

[Fig. 1] Environnement des villes antiques de Plinthine et Taposiris Magna qui fait l'objet des investigations de la MrFTM. Au fond à gauche, le lac Mariout (en cours d'assèchement) et, à droite, les vestiges du temple surplombant la ville de Taposiris Magna.

[Fig.2] Tombe 5o et vestiges du

monument à degrés la signalant.
[Fig.3] Vue axonométrique de la tombe 3 Le dromos de cette tombe permet aux proches de commémorer le défunt. Ce type de tombe rappelle les hypogées alexandrins, dont l'influence serait macédonienne.

[Fig.4] Décor d'un loculus en plâtre particulièrement connu, avec des caractères grecs (chapiteaux des colonnes) et égyptiens (Anubis). Après la conquête grécomacédonienne de l'Égypte, l'installation d'un nombre important d'étrangers, en majorité grecs ou de culture hellène, conduit à l'instauration d'une société multiculturelle.

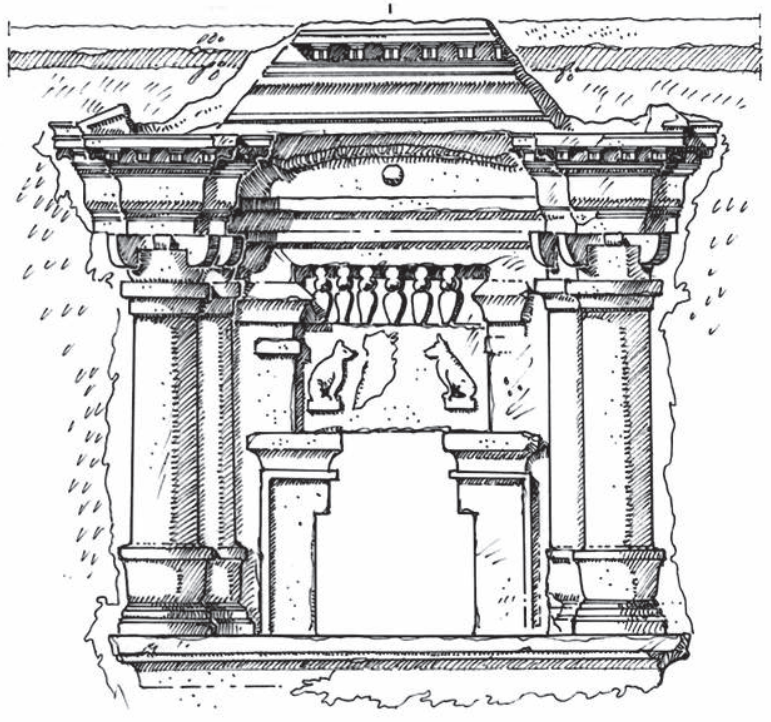


nous renvoie vers nos collections, qu'elle nous pousse à valoriser en les replaçant mieux dans leur contexte, nous pourrions faire bénéficier les Égyptiens de l'expérience française en ce domaine. Nous pourrions aussi leur faire partager notre savoir-faire en matière de protection et de valorisation des monuments historiques. Dans cette optique, Christophe Thiers, le nouveau codirecteur français du Centre franco-égyptien d'étude des temples de Karnak (CNRS-MAEE-CsA) vient de signer avec Mansour Boraïk, codirecteur égyptien du même centre, un accord concernant la formation d'inspecteurs du Conseil suprême des antiquités égyptiennes et celle de jeunes universitaires égyptiens. Les thèmes abordés dans ce chantier-école dérivent de l'étude scientifique des temples de Karnak menée lors des fouilles et autres chantiers conduits par le centre francoégyptien. Jean-Yves Empereur, directeur du Centre d'études alexandrines, fait de même, et son équipe est constituée d'un nombre important d'archéologues égyptien(ne)s.

Dans ces perspectives, la coopération archéologique franco-égyptienne est prometteuse. Par l'intermédiaire de l'ambassade de France au Caire et de missions françaises installées de façon permanente en Égypte, des archéologues de l'Inrap sont de plus en plus souvent sollicités. Ainsi, la mission du Louvre sur le site de Saqqarah va s'enrichir à l'automne 2010 de la collaboration d'un archéologue de l'Inrap, comme le fait déjà sur certains sites l'Institut français d'archéologie orientale du Caire. C'est reconnaître et valoriser la compétence des chercheurs de l'Inrap, leur performance dans l'enregistrement des données archéologiques et l'excellence des résultats qu'ils produisent. Souhaitons à l'archéologie égyptienne, dont la mission la plus urgente est d'être préventive, de voir s'activer des équipes mixtes, dédiées à cette tâche noble et prioritaire.

\section{La démarche archéo- anthropologique dans la nécropole hellénistique de Plinthine}

1 C'est le titre d'une exposition qui, en 1998 avait mis en lumière les vestiges antiques de cette ville et de ses environs.

2 En ce sens, les résultats sont encore plus maigres que dans les nécropoles alexandrine : cf. Dunand, 2002

3 Une opération dirigée par Rached Nouweir en 1954 a fait l'objet d'une courte publication l'anné suivante : Nouweir, 1955. Faisel el-Ashmawy a effectué des recherches de terrain en 1980 . La dernière mission égyptienne, dirigée par Ezet el-Hamamy, a eu lieu en 1982. Ces dernières missions sont inédites. Bref résumé dans Venit, 2002, p. 169-17o. et épargnées par l'urbanisation intensive du littoral méditerranéen : Taposiris Magna et Plinthine (Boussac, 2001 et 2005) [Fig.1]. Leur abandon sans réoccupation, vraisemblablement à la fin de la période hellénistique pour Plinthine et à la fin de l'Antiquité pour Taposiris, a assuré la préservation de leurs vestiges ; certains ont été décrits et figurés par les rares expéditions ayant longé la côte au XIX ${ }^{\mathrm{e}}$ siècle. Remarquées donc, mais éclipsées par la gloire d'Alexandrie ${ }^{\mathbf{1}}$, ces villes sont tombées dans l'oubli, malgré l'intérêt que leur ont porté quelques chercheurs. Elles illustrent pourtant l'urbanisme et les modes de vie des bourgades de la chôra alexandrine. C'est la raison pour laquelle, depuis 1998, la Mission française des fouilles de Taposiris Magna (MғFтM), dirigée par Marie-Françoise Boussac, a engagé l'étude de ces deux sites. Sous l'égide de la Maison de l'Orient Méditerranéen (Mom) et du ministère des Affaires étrangères et européennes, en collaboration avec l'Inrap et avec la participation du Conseil suprême des antiquités égyptiennes, il s'agit d'explorer ces deux cités et leur territoire au rythme annuel d'une mission de terrain.

Fouille ancienne et nouvelles perspectives archéo-thanatologiques pour la nécropole de Plinthine. Parmi les nombreux dossiers développés au sein de la Mfftm, la nécropole de Plinhine n'est pas des moindres. L'importance de cette nécropole pour l'étude des pratiques funéraires a été remarquée par de nombreux auteurs, notamment en raison de son état de conservation (Boussac et al., 2006). En 1937, A. Adriani mène une première campagne de fouilles un an après un pillage limité à quelques tombes. «Toutefois, écrit-il, les recherches que nous avons effectuées permettent un jugement sûr quant aux caractères et à la chronologie de la nécropole, qui (...) garde pour nous toute son importance documentaire pour l'étude des sites. » Il poursuit: « Pour la première fois, dans l'histoire des recherches dans la Maréotis, nous avons trouvé une nécropole de la haute époque hellénistique, ayant toutes les caractéristiques des nécropoles grecques de la métropole » (Adriani, 1952, p. 140). Cet ensemble, que le chercheur juge "vaste », est détaillé au sein d'une publication certes non exhaustive, mais qui autorise aujourd'hui quelques pistes de réflexion à la lumière des découvertes récentes. Les données intéressent essentiellement l'organisation de surface (enclos, monuments funéraires), l'architecture et les pratiques funéraires (inhumation et crémation) (Adriani, 1952) [Fig.2]. Ces dernières sont appréhendées en termes très généraux (cadavres, squelettes et urnes). Ainsi, les précisions concernant les squelettes, pourtant retrouvés par dizaines, sont rares ; aucun examen n'a été pratiqué sur les restes humains.

Le Service des antiquités égyptien a procédé par la suite à d'autres interventions, dans les années 1950 et $1980^{3}$. Chacune de ces fouilles a permis de mettre au jour, plus ou moins accidentellement, des tombes présentant des types très variés allant de la simple fosse (sépulture individuelle de surface)
Marie-Françoise Boussac
Université Paris X-Nanterre, UMR 5189 « Histoire et Sources des Mondes Antiques »

Inrap, UMR 5199 « De la Préhistoire à l'Actuel: Culture, Environnement, Anthropologie»

$\mathrm{D}$ ans l'Antiquité, à l'ouest d'Alexandrie s'étendait la Maréotide, une région prospère en raison de sa proximité avec la capitale des Lagides, mais pas seulement. Bordé au nord par la Méditerranée et baigné par le lac Maréotis au sud, l'endroit était particulièrement propice à la viticulture et au commerce. Un réseau dense de ports lacustres et de villas agricoles constituent les signes les plus évidents de cette situation.

C'est dans ce contexte que se situent deux villes antiques, implantées sur la rive nord du lac Maréotis 